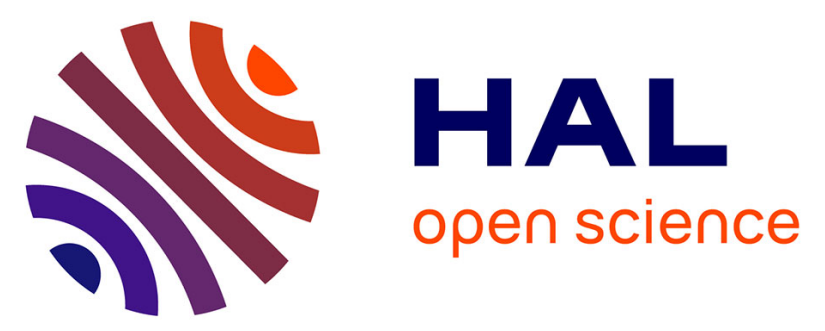

\title{
Polarization synchronization in unidirectionally coupled vertical-cavity surface-emitting lasers with orthogonal optical injection
}

\author{
M Sciamanna, I Gatare, A. Locquet, K Panajotov
}

\section{To cite this version:}

M Sciamanna, I Gatare, A. Locquet, K Panajotov. Polarization synchronization in unidirectionally coupled vertical-cavity surface-emitting lasers with orthogonal optical injection. Physical Review E: Statistical, Nonlinear, and Soft Matter Physics, 2007, 75, 10.1103/physreve.75.056213 . hal-02993526

\section{HAL Id: hal-02993526 \\ https://hal.science/hal-02993526}

Submitted on 6 Nov 2020

HAL is a multi-disciplinary open access archive for the deposit and dissemination of scientific research documents, whether they are published or not. The documents may come from teaching and research institutions in France or abroad, or from public or private research centers.
L'archive ouverte pluridisciplinaire HAL, est destinée au dépôt et à la diffusion de documents scientifiques de niveau recherche, publiés ou non, émanant des établissements d'enseignement et de recherche français ou étrangers, des laboratoires publics ou privés. 


\title{
Polarization synchronization in unidirectionally coupled vertical-cavity surface-emitting lasers with orthogonal optical injection
}

\author{
M. Sciamanna, ${ }^{1, *}$ I. Gatare, ${ }^{1,2, \dagger}$ A. Locquet, ${ }^{3, \ddagger}$ and K. Panajotov ${ }^{2, \S}$ \\ ${ }^{1}$ Supélec, LMOPS CNRS UMR-7132, Unité de Recherche Commune Supélec et Université de Metz, Rue Edouard Belin 2, \\ F-57070 Metz, France \\ ${ }^{2}$ Department of Applied Physics and Photonics, TW-TONA, Vrije Universiteit Brussel (VUB), B-1050 Brussels, Belgium \\ ${ }^{3}$ Unité Mixte Internationale 2958 Georgia Tech-CNRS, Georgia Tech Lorraine, 2 Rue Marconi, F-57070 Metz, France
}

(Received 13 November 2006; revised manuscript received 19 February 2007; published 22 May 2007)

\begin{abstract}
We analyze theoretically the polarization dynamics in unidirectionally coupled vertical-cavity surfaceemitting lasers (VCSELs). The master VCSEL is subject to an isotropic optical feedback. The slave VCSEL is subject to an orthogonal optical injection from the master VCSEL, i.e., only the linearly polarized mode orthogonal to the dominant linearly polarized mode of the free-running slave VCSEL is injected into the slave VCSEL. This laser configuration may lead the slave VCSEL polarization to switch to that of the injected master laser field. The injected power required for polarization switching depends on the frequency detuning. We identify in the plane of the injection parameters two regions of enhanced synchronization between the injected LP mode and the corresponding slave LP mode. In the so-called region II the slave VCSEL exhibits anticorrelated dynamics in its two LP modes while in the so-called region I the slave VCSEL exhibits dynamics in only one LP mode, which corresponds to the polarization of the injected field. The two regions exhibit different synchronization properties in both the LP mode dynamics and total intensity dynamics. We furthermore analyze the dependency of the synchronization quality on the parameter mismatch between master and slave VCSELs and on the polarization switching properties of each VCSEL.
\end{abstract}

DOI: 10.1103/PhysRevE.75.056213

PACS number(s): 05.45.- a, 42.65.Sf

\section{INTRODUCTION}

Vertical-cavity surface-emitting lasers (VCSELs) exhibit several desirable characteristics such as a small threshold current, circular beam profile with narrow divergence, single longitudinal mode emission, wafer-scale testing and array capabilities [1]. These great advantages have stimulated their development and use in applications previously reserved for conventional edge-emitting lasers. VCSELs have also attracted considerable attention in reason of their complex polarization properties: the emitted light is typically linearly polarized (LP) along one of two preferential and orthogonal directions $(x$ and $y)$ but the light polarization may easily switch between these two $x$ and $y$ LP modes as the laser operating conditions such as current or temperature vary $[2,3]$. The so-called polarization switching has been the subject of several theoretical and experimental studies for more than 10 years, both for the understanding of the underlying physical mechanism and for the development of polarization control techniques in polarization-sensitive applications; for a review see, for example, Refs. [4,5]. Additional complexities in the VCSEL polarization dynamics arise when the laser is subject to delayed back-reflection of the emitted light (op-

\footnotetext{
*Electronic address: marc.sciamanna@supelec.fr. Also at Unité Mixte Internationale 2958 Georgia Tech-CNRS, Georgia Tech Lorraine, 2 Rue Marconi, F-57070 Metz, France.

†Electronic address: gatarega_ign@ metz.supelec.fr

*Electronic address: alocquet@georgiatech-metz.fr

${ }^{\S}$ Electronic address: kpanajotov@tona.vub.ac.be. Also at Institute of Solid State Physics, 72 Tzarigradsko Chaussee Blvd., 1784 Sofia, Bulgaria.
}

tical feedback) [6-8], to optical injection from a second laser [9], or to large current modulation in data communications $[10,11]$. These laser configurations can destabilize the VCSEL dynamics into complex time-periodic, quasiperiodic or even chaotic dynamics [12-16], or control the light polarization and lock the VCSEL into a well-defined steady-state polarization dynamics $[9,17-20]$ or stable polarization selfmodulation [21-24].

Chaotic VCSELs are highly desirable compact light sources that can be used in chaos-based secure communications [25-28]. The idea of chaotic secure communications is to encode a message into a noiselike chaotic emitter and to decode the message by using a receiver that is synchronized to the chaos generated by the emitter. Chaos synchronization has been demonstrated in several laser systems, including $\mathrm{Nd}: Y A G$ [29], $\mathrm{CO}_{2}$ [30], fiber lasers [26,27], and semiconductor edge-emitting lasers [31-33]. Experimental demonstration of message encoding-decoding has been shown by several groups and a state-of-the-art report has been given recently by Argyris et al. [34]. By contrast, studies on VCSEL synchronization remain scarce. First theoretical studies by Spencer et al. [35,36] have unveiled the possibility to synchronize chaos in coupled VCSELs in master-slave configuration. However, they did not take into account the complex polarization dynamics of VCSELs. Additional more recent theoretical investigations have modeled synchronized coupled VCSELs, however focusing mostly on the total intensity synchronization properties [37]. Very recently, first experiments have demonstrated chaos synchronization in VCSELs in either master-slave unidirectional configuration [38] or in mutually coupled configuration [39]. The practical use of chaos synchronized VCSELs for secure message transmission has also been tested recently in laboratory [40], 
with successful encoding-decoding of a $200 \mathrm{MHz}$ sinusoidal message. These first experimental studies motivate a new, detailed theoretical analysis of chaos synchronization in coupled VCSELs, which would take into account the complex polarization switching properties and polarization dynamics in VCSELs.

In this paper, we analyze theoretically the synchronization properties and polarization dynamics of two VCSELs that are unidirectionally coupled in a master-slave configuration that has been recently investigated experimentally by Hong et al. [38] and Lee et al. [40] The configuration is such that (1) the master VCSEL is rendered chaotic via optical feedback, (2) the master laser exhibits chaos in its two orthogonal $x$ - and $y$-LP modes, (3) the $x$-LP mode of the chaotic emitter is rotated to $y$-LP direction and injected into the slave laser, (4) the free-running slave VCSEL emits almost only the $x$-LP mode, the $y$-LP mode being strongly suppressed, and finally (5) the slave laser is only subject to optical injection and not to optical feedback (such a configuration is also called "open-loop" [41]). The coupled VCSEL configuration is therefore based on an isotropic optical feedback on the master laser, and an orthogonal optical injection on the slave laser. It is worth mentioning that, besides its applications in VCSEL experiments, a configuration based on polarizationrotated optical injection has also recently received interest in coupled edge-emitting lasers to demonstrate identity synchronization [42].

Our theoretical analysis successfully reproduces qualitatively the VCSEL experimental results, that is, coupling light from the master to the slave VCSEL makes it possible to excite the normally depressed $y$-LP mode in the slave laser and to induce a good synchronization between the $y$-LP mode dynamics of the slave laser and the chaotic dynamics in the injected LP mode of the master laser. The chaos synchronization between $y$-LP slave laser mode and $x$-LP injected master laser mode is furthermore accompanied by an antisynchronization between the $x$-LP slave laser mode and the $x$-LP injected master laser mode. The antisynchronization is the result of the anticorrelated dynamics between LP modes in the slave laser. Moreover, our theoretical results unveil features that will motivate additional investigations.

Our paper is organized as follows. In Sec. II we detail the rate equation model that has been used for our theoretical analysis of the coupled VCSEL scheme. Section III reports on synchronization properties and polarization dynamics. Two different synchronization mechanisms are reported, and they are mapped in the plane of the injection parameters (injection rate vs. frequency detuning) in Sec. IV. Section IV also analyzes the dependency of the synchronization properties on parameter mismatch between the VCSELs. Section V analyzes the robustness of the synchronization result against modifications of the spin-flip relaxation rate, which is an important but often unknown parameter of the VCSEL model. Conclusions are presented in Sec. VI.

\section{RATE EQUATION MODEL}

The polarization dynamics in each of the two coupled VCSELs is modeled by an extension of the spin-flip model
(SFM) [43] that accounts for cavity anisotropies, for the isotropic optical feedback in the master laser equations, and for an orthogonal optical injection in the slave laser equations. The parameters of the slave laser are adjusted in such a way that it emits $x$-LP light when not coupled. The $x$-LP mode of the master laser is then rotated to the $y$ direction before being injected into the slave laser, i.e., the polarization of the coupled light is parallel to the suppressed LP mode of the slave laser. Our rate equations are

$$
\begin{gathered}
\frac{d F_{x_{M}}}{d t}=\kappa(1+i \alpha)\left(D_{M} F_{x_{M}}+i d_{M} F_{y_{M}}-F_{x_{M}}\right)-i \gamma_{p_{M}} F_{x_{M}} \\
-\gamma_{a_{M}} F_{x_{M}}+f F_{x_{M}}(t-\tau) \exp \left(-i \omega_{M} \tau\right), \\
\frac{d F_{y_{M}}}{d t}=\kappa(1+i \alpha)\left(D_{M} F_{y_{M}}-i d_{M} F_{x_{M}}-F_{y_{M}}\right)+i \gamma_{p_{M}} F_{y_{M}} \\
+\gamma_{a_{M}} F_{y_{M}}+f F_{y_{M}}(t-\tau) \exp \left(-i \omega_{M} \tau\right), \\
\frac{d D_{M}}{d t}=-\gamma_{e}\left[D_{M}\left(1+\left|F_{x_{M}}\right|^{2}+\mid F_{y_{M}}{ }^{2}\right)\right]+\gamma_{e} \mu_{M} \\
-i \gamma_{e} d_{M}\left(F_{y_{M}} F_{x_{M}}^{*}-F_{x_{M}} F_{y_{M}}^{*}\right), \\
\frac{d d_{M}}{d t}=-\gamma_{s} d_{M}-\gamma_{e} d_{M}\left(\left|F_{x_{M}}\right|^{2}+\left|F_{y_{M}}\right|^{2}\right) \\
\quad-i \gamma_{e} D_{M}\left(F_{y_{M}} F_{x_{M}}^{*}-F_{x_{M}} F_{y_{M}}^{*}\right)
\end{gathered}
$$

for the master laser, and for the slave laser

$$
\frac{d F_{x_{S}}}{d t}=\kappa(1+i \alpha)\left(D_{S} F_{x_{S}}+i d_{S} F_{y_{S}}-F_{x_{S}}\right)-i \gamma_{p_{S}} F_{x_{S}}-\gamma_{a_{S}} F_{x_{S}},
$$

$$
\begin{gathered}
\frac{d F_{y_{S}}}{d t}=\kappa(1+i \alpha)\left(D_{S} F_{y_{S}}-i d_{S} F_{x_{S}}-F_{y_{S}}\right)+i \gamma_{p_{S}} F_{y_{S}}+\gamma_{a_{S}} F_{y_{S}} \\
+\eta F_{x_{M}}\left(t-\tau_{c}\right) \exp \left[i\left(\omega_{M}-\omega_{S}\right) t\right] \exp \left(-i \omega_{M} \tau_{c}\right) \\
\frac{d D_{S}}{d t}=-\gamma_{e}\left[D_{S}\left(1+\left|F_{x_{S}}\right|^{2}+\mid F_{y_{S}}{ }^{2}\right)\right]+\gamma_{e} \mu_{S} \\
-i \gamma_{e} d_{S}\left(F_{y_{S}} F_{x_{S}}^{*}-F_{x_{S}} F_{y_{S}}^{*}\right),
\end{gathered}
$$

$$
\frac{d d_{S}}{d t}=-\gamma_{S} d_{S}-\gamma_{e} d_{S}\left(\left|F_{x_{S}}\right|^{2}+\mid F_{y_{S}}{ }^{2}\right)-i \gamma_{e} D_{S}\left(F_{y_{S}} F_{x_{S}}^{*}-F_{x_{S}} F_{y_{S}}^{*}\right) .
$$

$F_{x, y}$ are the two linearly polarized slowly varying components of the field and $D$ and $d$ are two carrier variables. $D$ accounts for the total population inversion between conduction and valence bands, while $d$ is the difference between population inversions for the spin-up and spin-down radiation channels. The internal VCSEL parameters are as follows: $\kappa$ is the field decay rate, $\gamma_{e}$ is the decay rate of $D, \gamma_{s}$ is the spin-flip relaxation rate (which accounts for the different 


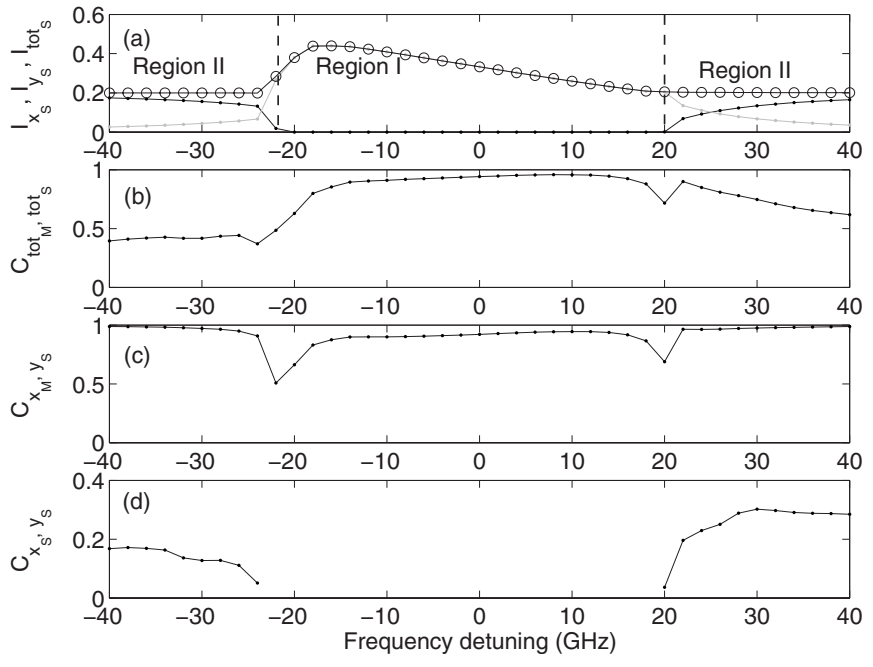

FIG. 1. Evolution of the time-averaged LP mode and total intensities in the slave laser (a), of the correlation coefficient $C_{\text {tot }_{M}, \text { tot }_{S}}$ (b), $C_{x_{M}, y_{S}}$ (c), $C_{x_{S}, y_{S}}$ (d) as function of the frequency detuning, for a fixed injection rate $\eta=100 \mathrm{GHz}$. Parameters are specified in the text.

microscopic mechanisms involved in the homogenization of the carrier spins), $\alpha$ is the linewidth enhancement factor, $\mu$ is the normalized injection current ( $\mu=1$ at threshold), $\gamma_{a}$ is the linear dichroism, and $\gamma_{p}$ is the linear birefringence. The isotropic optical feedback in the master laser equations (5)-(8) has been included in the same way as in Ref. [12], with $\omega_{M}$ being the optical frequency of the $x$ - and $y$-polarized modes at the solitary laser threshold in the absence of linear anisotropies. The parameter $f$ is the feedback rate, $\tau$ is the external cavity delay time, $\tau_{c}$ is the propagation delay time between the coupled VCSELs, $\eta$ is the injection rate. The detuning is defined as $\Delta \omega=\omega_{M}-\omega_{S}$, where $\omega_{S}$ is the slave optical frequency and is defined in the same way as $\omega_{M}$. We consider first a case for which all parameters of the master and slave lasers are identical, except for the laser frequencies. We take the following values for the laser parameters: $\gamma_{e}=1 \mathrm{~ns}^{-1}, \kappa=300 \mathrm{~ns}^{-1}, \alpha=3, f=5 \mathrm{GHz}, \tau_{c}=0, \gamma_{s}=50 \mathrm{~ns}^{-1}$, $\omega_{M} \tau=6 \mathrm{rad}, \tau=3 \mathrm{~ns}, \gamma_{a_{S}}=\gamma_{a_{M}}=-0.1, \gamma_{p_{S}}=\gamma_{p_{M}}=8.5 \mathrm{rad} / \mathrm{ns}$, and $\mu_{M}=\mu_{S}=1.2$. With these values of the VCSEL parameters the free-running lasers exhibit a single $x$-LP mode stationary dynamics.

\section{POLARIZATION SWITCHING AND POLARIZATION SYNCHRONIZATION}

As a result of the optical feedback, the master laser dynamics exhibits optical chaos in its two LP modes. The master laser $x$-LP mode is then rotated to orthogonal direction and injected into the slave VCSEL. We show in Fig. 1(a) the evolution of the averaged intensities in the two LP modes of the slave laser (with dots) and of the slave laser total intensity (with circles) as we vary the frequency detuning between master and slave lasers and for a fixed value of the injected power $\eta=100 \mathrm{GHz}$. As we scan the frequency detuning from negative to positive detuning values we can observe a polarization switching from the normally dominant $x$-LP slave laser mode to the normally suppressed $y$-LP slave laser mode and then back to the $x$-LP mode. We find therefore a detuning interval inside which the slave laser switches its polarization to that of the injected light, and moreover inside which the normally dominant slave laser $x$-LP mode is fully suppressed. We shall call this region "region I" to emphasize that the slave VCSEL only exhibits a single-mode dynamics (in the $y$-LP mode). As shown in Fig. 1(a) the region I expands from frequency detuning of $-22 \mathrm{GHz}$ up to about $20 \mathrm{GHz}$. Outside region I the slave VCSEL exhibits dynamics in its two LP modes, and therefore we shall call this region "region II." In region I the total intensity emitted by the slave VCSEL is larger than in region II and depends on the frequency detuning. In region II the total intensity of the slave laser light is kept constant whatever the frequency detuning, which relates to the fact that the intensity in the $x$-LP mode decreases at the benefit of the increase in the $y$-LP mode intensity. In region I the total intensity of the slave laser light increases because the slave laser mode emits only in the $y$-LP mode and the injected light therefore interacts coherently with the slave $y$-LP mode and supports the $y$-LP mode intensity. In region II the slave laser dynamics consists of a dominant $x$-LP mode dynamics interacting incoherently with the injected light and, hence, the slave total intensity is purely the incoherent addition of its two LP mode intensities.

An interesting question, motivated by previous studies on chaos synchronization in coupled edge-emitting lasers, is whether the chaotic dynamics of the slave laser, induced by the optical injection, is able to synchronize to the master laser optical chaos. We find that indeed not only the LP mode dynamics but possibly also the total intensity dynamics can be synchronized between master and slave lasers, depending on the injection strength and frequency detuning. In the following we analyze quantitatively the synchronization quality between the intensity time traces by using the following cross-correlation coefficients $C_{x_{M}, y_{S}}, C_{x_{S}, y_{S}}$, and $C_{\text {tot }_{M}, \text { tot }_{S}}$ :

$$
\begin{gathered}
C_{x_{M}, y_{S}}=\frac{\left\langle\left[I_{x_{M}}\left(t-\tau_{c}\right)-\left\langle I_{x_{M}}\right\rangle\right]\left[I_{y_{S}}(t)-\left\langle I_{y_{S}}\right\rangle\right]\right\rangle}{\sqrt{\left\langle\left[I_{x_{M}}(t)-\left\langle I_{x_{M}}\right\rangle\right]^{2}\right\rangle\left\langle\left[I_{y_{S}}(t)-\left\langle I_{y_{S}}\right\rangle\right]^{2}\right\rangle}}, \\
C_{x_{S}, y_{S}}=\frac{\left\langle\left[I_{x_{S}}\left(t-\tau_{c}\right)-\left\langle I_{x_{S}}\right\rangle\right]\left[I_{y_{S}}(t)-\left\langle I_{y_{S}}\right\rangle\right]\right\rangle}{\sqrt{\left\langle\left[I_{x_{S}}(t)-\left\langle I_{x_{S}}\right\rangle\right]^{2}\right\rangle\left\langle\left[I_{y_{S}}(t)-\left\langle I_{y_{S}}\right\rangle\right]^{2}\right\rangle}}, \\
C_{\text {tot }_{M}, \text { tot }_{S}=}=\frac{\left\langle\left[I_{\text {tot }_{M}}\left(t-\tau_{c}\right)-\left\langle I_{\text {tot }_{M}}\right\rangle\right]\left[I_{\text {tot }_{S}}(t)-\left\langle I_{\text {tot }_{S}}\right\rangle\right]\right\rangle}{\sqrt{\left\langle\left[I_{\text {tot }_{M}}(t)-\left\langle I_{\text {tot }_{M}}\right\rangle\right]^{2}\right\rangle\left\langle\left[I_{\text {tot }_{S}}(t)-\left\langle I_{\text {tot }_{S}}\right\rangle\right]^{2}\right\rangle}} .
\end{gathered}
$$

For the coupling configuration considered here, we only observe synchronization of the isochronous type [44-47], which leads to a maximum of the correlation for a time lag equal to $\tau_{c}$ between the intensity time traces. In our case we have chosen $\tau_{c}=0$. Anticipating synchronization $[45,48,49]$ is not observed, as could be expected for such a dissymmetric coupling in which we have orthogonal injection of only one of the two LP components.

Figure 1 shows the correlation degree between the time traces of the total intensity in master and slave lasers (b), the 

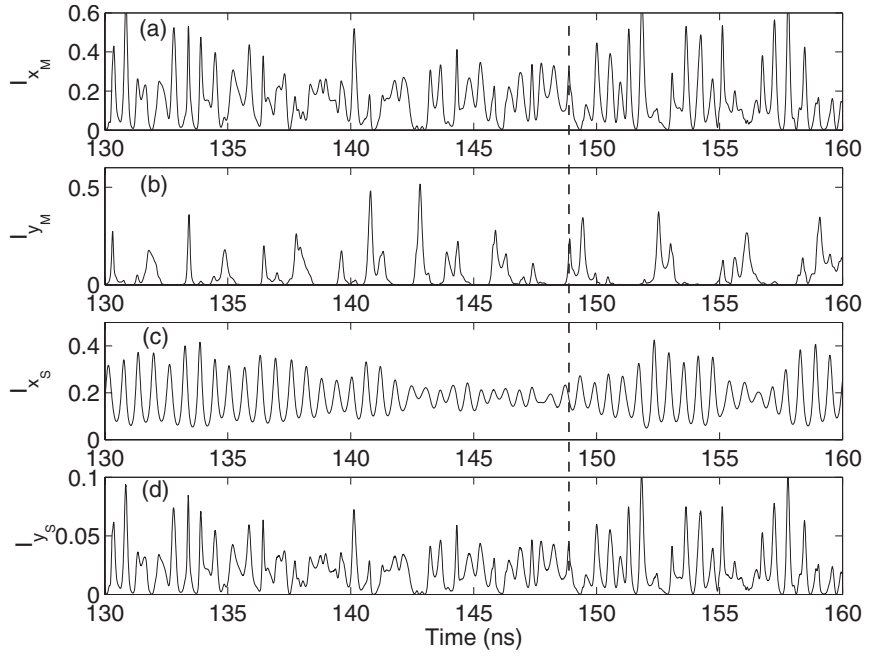

FIG. 2. Time series of master laser and slave laser LP mode intensities, for $\eta=100 \mathrm{GHz}$ and $\Delta \omega=-40 \mathrm{GHz}$ (corresponding to region II).

$x$-LP master laser intensity and the $y$-LP slave laser intensity (c), and between the intensities of the slave laser modes (d), as we scan the frequency detuning and for a fixed injection strength. Interestingly, Fig. 1(b) shows that the total intensities in master and slave lasers are badly synchronized in region II (correlation coefficient very small) even though the correlation coefficient $C_{x_{M}, y_{S}}$ [in Fig. 1(c)] is close to 1, which implies an almost perfect synchronization between the $x$-LP injected light intensity dynamics and the dynamics in the slave laser $y$-LP mode. By contrast, in region I the total intensities of the master and slave reveal a degree of synchronization similar to that existing between the master $x$-LP and slave $y$-LP modal intensities, with a maximum correlation coefficient around 0.9. Even though, for the parameter set considered here, the synchronization of the total intensities is better in region I than in region II, the synchronization quality between the master $x$-LP and the slave $y$-LP components is better in region II than in region I. This outlines the importance of considering polarization-resolved synchronization. The analysis of the correlation between slave LP mode intensities in Fig. 1(d) does not provide clear indication of correlated or anticorrelated dynamics, since the computed correlation coefficient is very close to zero. The cross correlation $C_{x_{S}, y_{S}}$ is not computed in region I since there the $x$-LP slave laser mode is fully suppressed.

Figure 2 analyzes in more detail the time traces of master and slave laser LP intensities, in the synchronization conditions corresponding to region II $(\eta=100 \mathrm{GHz}$ and $\Delta \omega / 2 \pi$ $=-40 \mathrm{GHz})$. The comparison between the time traces in (a) and (d) shows that the intensity corresponding to the depressed $y$-LP mode of the slave laser is almost perfectly synchronized with the injected $x$-LP intensity of the master laser, with a time-lag equal to $\tau_{c}=0$ between the intensity time traces (as better seen with the reference dashed line). The comparison between the time traces (a) and (b), i.e., the time traces of intensities in the LP master laser modes, shows that the LP mode intensities exhibit fast in-phase pulsing but also anticorrelated dynamics on a slower time scale: when one
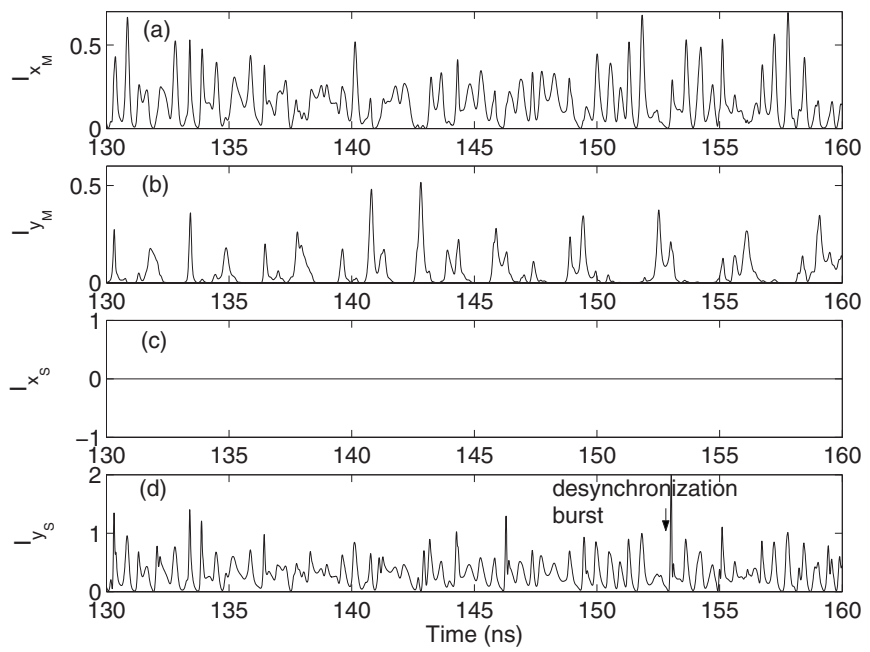

FIG. 3. Time series of master laser and slave laser LP mode intensities, for $\eta=100 \mathrm{GHz}$ and $\Delta \omega=0 \mathrm{GHz}$ (corresponding to region I).

mode is dominant, the other mode is depressed and vice versa. Such a mode competition with in-phase fast pulsing when the two modes are excited together has been already observed theoretically and experimentally in VCSELs subject to optical feedback $[15,16]$. The comparison between the time traces (c) and (d), i.e., the time traces of intensities in the LP slave laser modes, shows no clear indication of correlated or anticorrelated dynamics on the pulsing time scale, which corresponds to the close to zero correlation coefficient plotted in Fig. 1(d).

Figure 3 is the same as Fig. 2 but for different coupling conditions $(\eta=100 \mathrm{GHz}$ and $\Delta \omega / 2 \pi=0 \mathrm{GHz})$, corresponding to region I. As shown in panel (c), in this region the slave laser $x$-LP mode is fully depressed and the slave VCSEL switches its polarization from a normally dominant $x$-LP light polarization to a now dominant $y$-LP light polarization. The comparison between the time traces in (a) and (d) shows that the slave laser $y$-LP intensity dynamics is relatively well synchronized with the injected master laser $x$-LP intensity dynamics. We observe, however, desynchronization bursts in particular during short time-periods following the switch-off of the injected light. The arrow in Fig. 3(d) indicates such a desynchronization burst following the switch off of the $x$-LP master laser intensity: the $y$-LP slave laser mode intensity [in (d)] exhibits a large pulse before recovering similar pulsing dynamics as in the injected one [in (a)]. It should also be noticed that the slave $y$-LP intensity is significantly larger than that of the master laser $x$-LP intensity, as already illustrated in Fig. 1(a). As a result of the partial loss of synchronization, the correlation coefficient taken on average on a long time trace is smaller than that computed for the case of region II in Fig. 2. As for region II, the synchronization occurs with a time-lag equal to $\tau_{c}=0$, as better seen with the reference dashed line.

In order to further complement the time-series analysis and to emphasize the qualitatively different synchronization properties of regions I and II, we show in Figs. 4(a1), (a2) the synchronization diagrams corresponding to Fig. 2 and in (b1), (b2) the synchronization diagrams corresponding to 

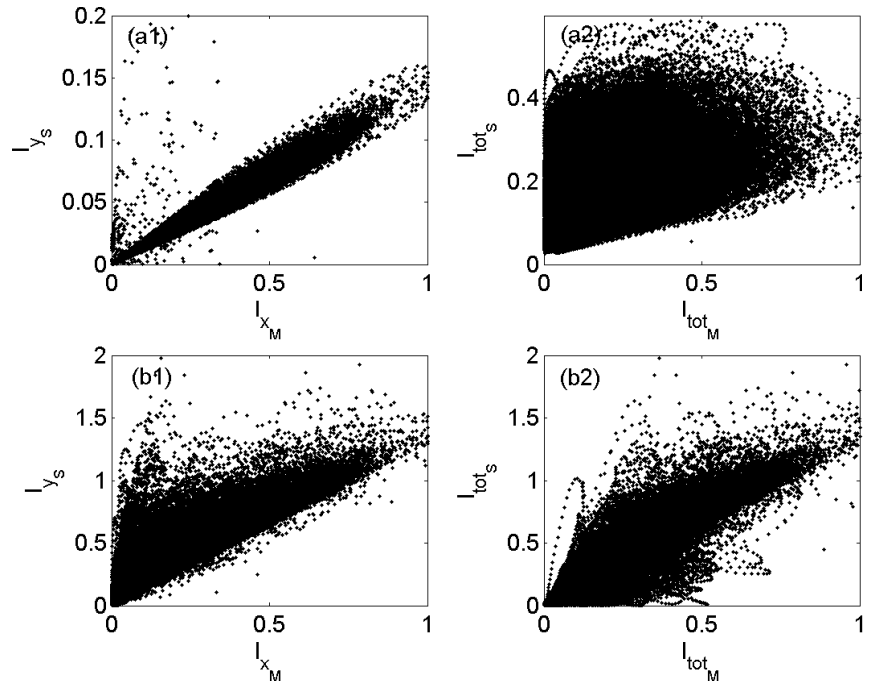

FIG. 4. Synchronization diagrams corresponding to regions II $[(\mathrm{a} 1),(\mathrm{a} 2)]$ and I [(b1), (b2)] of qualitatively different synchronization properties: (a1), (b1) $I_{y_{S}}$ as function of $I_{x_{M}}$, (a2), (b2) $I_{\text {tot }_{S}}$ as function of $I_{\text {tot }_{M}}$.

Fig. 3. In (a1), (b1) is plotted the intensity of the slave laser $y$-LP mode as a function of the intensity of the injected master $x$-LP mode; in (a2), (b2) is plotted the slave laser total intensity as a function of the master laser total intensity. In the case of injection parameters corresponding to region II, the slave $y$-LP mode intensity is almost perfectly synchronized with the injected $x$-LP mode intensity of the master laser, but with the slave laser synchronized $y$-LP mode intensity much smaller than the injected master $x$-LP mode intensity. However, the total intensities of the two lasers are almost uncorrelated, as shown in Fig. 4(a2) and also pointed out by the computation of the correlation coefficient in Fig. 1(b). The fact that the slave laser total intensity is badly synchronized to the master laser total intensity can be attributed to the fact that only the weak LP mode of the slave laser synchronizes very well with the dominant master LP mode. Therefore the contribution of the synchronized slave LP mode dynamics to the slave total intensity dynamics is very small. The synchronization diagram of Fig. 4(b1) better shows the desynchronization bursts between the $y$-LP slave laser intensity dynamics and the $x$-LP master laser intensity dynamics. In spite of these desynchronization events, the two LP mode intensity dynamics are relatively well synchronized in region I but the synchronization quality is clearly smaller than that observed in region II [see the comparison between (b1) and (a1)]. Moreover, the comparison between the synchronization diagram of Fig. 4(b2) and that of Fig. 4(a2) shows that in region I the total intensity dynamics are much better synchronized than in region II, as it was already clear from the computation of the correlation coefficient in Fig. 1(b).

The previous figures have considered a case of unidirectionally coupled VCSELs but where the two coupled lasers have the same internal parameters, in particular the same linear cavity anisotropies and injection currents. However, it is commonly observed in VCSEL experiments that the polarization switching and polarization dynamical properties are

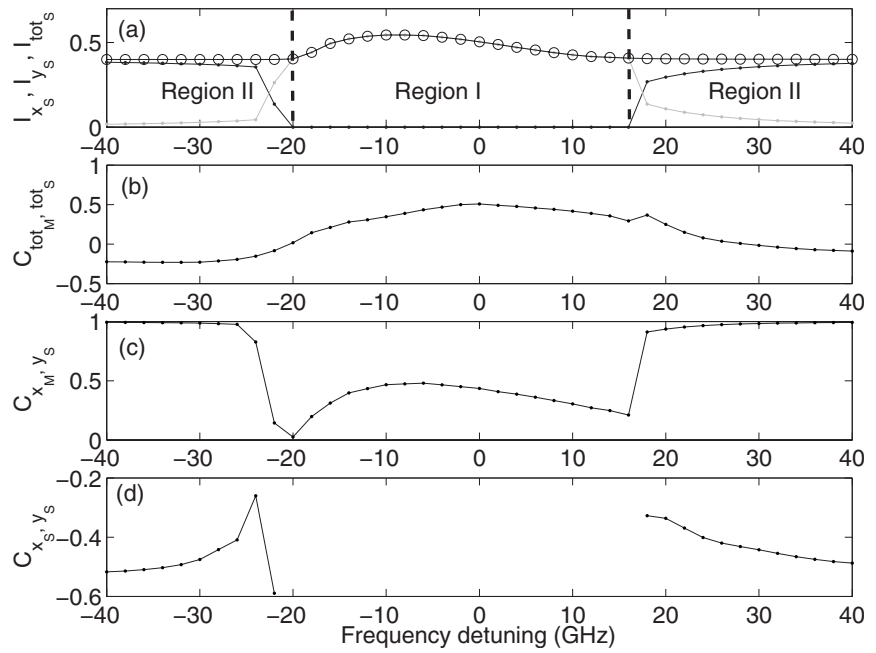

FIG. 5. Same as Fig. 1 but considering mismatch in VCSEL linear cavity anisotropies and injection currents: $\gamma_{a_{M}}=0.1, \gamma_{p_{M}}$ $=6 \mathrm{rad} / \mathrm{ns}, \mu_{M}=1.2$ and $\gamma_{a_{S}}=-0.1, \gamma_{p_{S}}=8.5 \mathrm{rad} / \mathrm{ns}, \mu_{S}=1.4$.

strongly dependent on the device linear anisotropies and operating conditions such as injection current or temperature [50]. We therefore consider now different values of the linear anisotropies $\gamma_{a}$ and $\gamma_{p}$ for the master and slave VCSELs, as well as different values of the injection current $\mu$. The VCSEL parameters and operating conditions are chosen such that the polarization properties are qualitatively similar to those used in the recent experiment of Ref. [38]. The master laser is simulated with $\gamma_{a_{M}}=0.1, \gamma_{p_{M}}=6 \mathrm{rad} / \mathrm{ns}$ and $\mu_{M}$ $=1.2$. The master laser exhibits a polarization switching from $x$-LP to $y$-LP mode at about $\mu \sim 1.3$ with bistability. The slave laser is simulated with $\gamma_{a_{S}}=-0.1, \gamma_{p_{S}}=8.5 \mathrm{rad} / \mathrm{ns}$, and $\mu_{S}=1.4$. The slave laser exhibits a single $x$-LP mode up to about $\mu=1$.6. In order to analyze the effect of the parameter mismatch on the polarization and total intensity synchronization properties, we plot in Fig. 5 the evolution of the LP mode and total output intensities and the correlation coefficients $C_{x_{M}, y_{S}}, C_{x_{S}, y_{S}}$, and $C_{\text {tot }_{M}, \text { tot }_{S}}$ as we scan the frequency detuning for a fixed injection strength, all other parameters remaining the same as those used for Fig. 1. We still observe in Fig. 5 the existence of two regions of qualitatively different polarization and synchronization properties. In region I, the slave has switched its polarization to that of the injected field and emits only the corresponding $y$-LP mode. In region II the slave laser exhibits a two-LP mode dynamics. Region II is characterized by an almost perfect synchronization between the $y$-LP slave intensity and the injected $x$-LP master intensity [see Fig. 5(c)], with a correlation coefficient very close to 1 . However in region II the total intensities of both master and slave VCSELs are almost uncorrelated [see Fig. $5(\mathrm{~b})$. In region I the synchronization between $y$-LP slave laser intensity and $x$-LP master laser intensity is much weaker than that observed in region II, with a maximum correlation coefficient close to 0.5 . In region I the total intensities of the two VCSELs synchronize as badly as do the corresponding polarization modes (maximum correlation coefficient close to 0.5 ), however the synchronization between total intensities is much better than the one observed in re- 
gion II. The inclusion of mismatch between the device parameters has strongly influenced the synchronization quality inside region I since, by comparing Fig. 1(b) and Fig. 5(b) [or Fig. 1(c) and Fig. 5(c)], we see that the correlation coefficients $C_{\mathrm{tot}_{M}, \mathrm{tot}_{S}}\left(\right.$ or $C_{x_{M}, y_{S}}$ ) have decreased from about a maximum of 0.9 to about a maximum of 0.5 . However, and interestingly, the synchronization quality in region II between $y$-LP slave laser mode and injected $x$-LP mode has not decreased when accounting for parameter mismatch: the comparison between Fig. 1(c) and Fig. 5(c) in region II shows a correlation coefficient $C_{x_{M}, y_{S}}$ still close to 1 . We also notice from Figs. 5(b) and 5(c) that the maximum correlation does not occur for zero detuning but is slightly shifted towards negative detuning values. As already mentioned in other laser configurations and laser systems [41], and as will be explained further in Sec. IV, this result is the consequence of the linewidth enhancement factor $\alpha$ which influences the injection-locking mechanism. Additionally, comparing Figs. 5(d) and 1(d), we observe that the cross-correlation coefficient $C_{x_{S}, y_{S}}$ between the slave laser mode intensities is now negative and larger in absolute value. The correlation coefficient close to -0.5 indicates a tendency for anticorrelation between the slave laser mode intensities. Our observation of synchronization of the injected light with the slave mode parallel to it and of therefore (weaker) antisynchronization with the orthogonal slave mode, qualitatively agrees with the experimental observations of Ref. [38]. On the basis of our study, we conjecture that the experimental conditions were such that the slave laser was operating in region II. The set of parameters leading to Fig. 5 being the most representative of the realistic experimental conditions of Ref. [38], we keep these parameters fixed for the numerical simulations performed in the remainder of this paper.

\section{MAPPING OF CORRELATION COEFFICIENT}

The preceding section has unveiled two synchronization mechanisms in our coupled VCSEL problem. One corresponds to region I in which the normally depressed $y$-LP slave laser mode synchronizes with the injected $x$-LP master laser mode, while the slave $x$-LP mode is completely suppressed by the orthogonal injection. The second corresponds to region II in which the slave laser exhibits a two-mode dynamics, with a depressed $y$-LP mode almost perfectly synchronized with the injected $x$-LP master laser mode and with anticorrelation between the slave laser LP mode dynamics.

We analyze in Figs. 6 and 7 the dependency of the $C_{x_{M}, y_{S}}$ and $C_{x_{S}, y_{S}}$ correlation coefficients, respectively, on the coupling parameters: injection rate $\eta$ and frequency detuning $\Delta \omega / 2 \pi$. A color map is used in each case and plotted on the right of the figure. The maximum injection rate is chosen such that it corresponds to a realistic value for VCSEL devices. Indeed we can write $\eta=r\left(1-r^{2}\right) t_{\mathrm{ext}} /\left(r \tau_{\mathrm{in}}\right)$, where $r$ is the amplitude reflectivity of the VCSEL output mirror, $t_{\mathrm{ext}}$ is the amplitude transmissivity in the path between the coupled VCSELs, and $\tau_{\text {in }}$ is the intracavity round-trip time. In the optimal case for which there is no attenuation in the path between coupled VCSELs, i.e., $t_{\mathrm{ext}}=1$, taking $r=0.995$ and

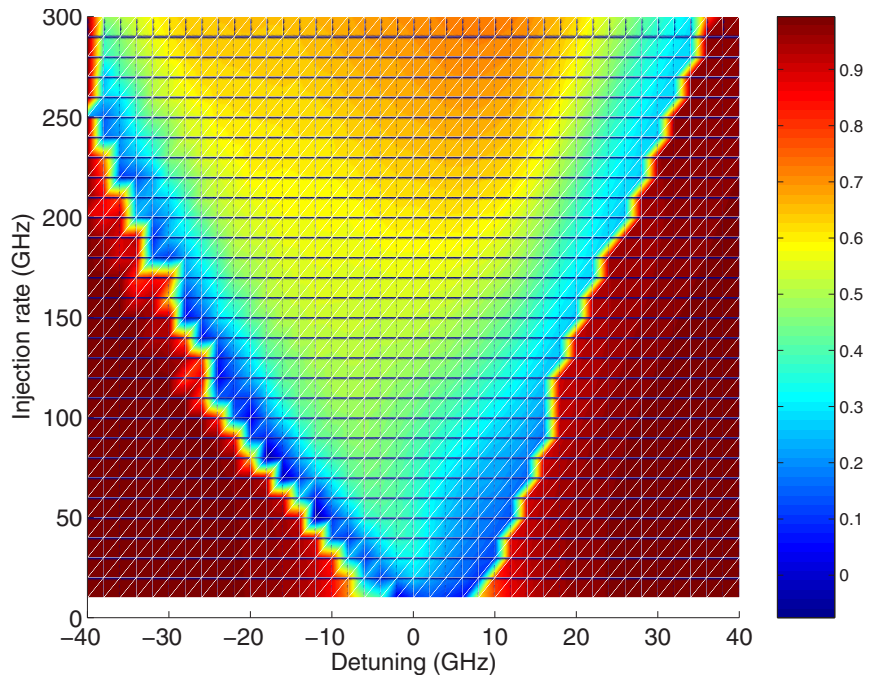

FIG. 6. (Color online) Mapping of correlation coefficient $C_{x_{M}, y_{S}}$ at zero time lag between the time traces corresponding to master $x$-LP mode and to slave $y$-LP mode, in the plane of the injection parameters.

$\tau_{\text {in }}=3 \times 10^{-5} \mathrm{~ns}$ as typical values for VCSELs [17] gives $\eta \sim 330 \mathrm{GHz}$.

As shown in Fig. 6, region I is approximately centered around zero frequency detuning and its boundaries span larger frequency detunings as we increase the injection strength. This region I corresponds to a relatively good synchronization quality, as the correlation coefficient can be as large as 0.8 for sufficiently large injection strengths. In this region, an injection locking-type of synchronization occurs, which is induced by the increase of the injected field strength [51]. The synchronization therefore increases in quality with the increase of the injection rate, and is progressively lost when the detuning is increased. By contrast region II is found for either large positive or negative detunings, with a boundary that depends on the injection strength. Region II

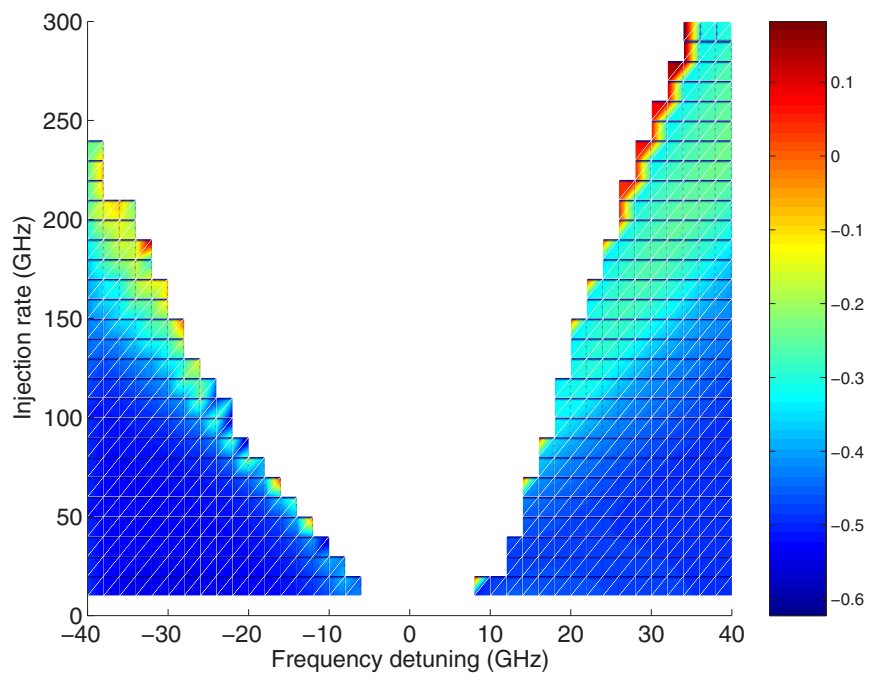

FIG. 7. (Color online) Same as Fig. 6 but showing the crosscorrelation coefficient $C_{x_{S}, y_{S}}$ between slave laser LP mode intensities. 


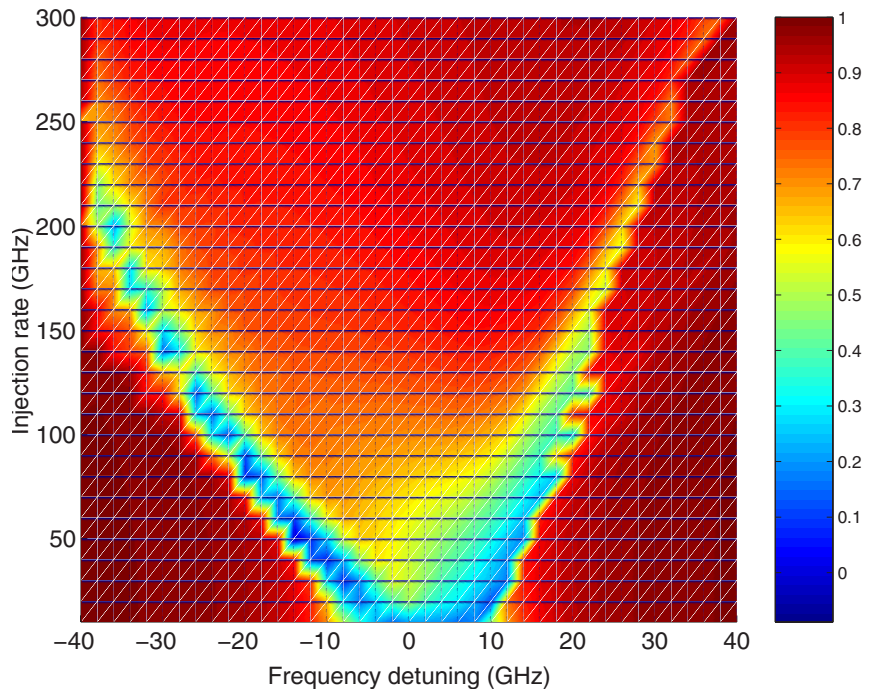

FIG. 8. (Color online) Same as Fig. 6 but with no mismatch between the values of linear cavity anisotropies in slave and master VCSELs.

corresponds to an almost perfect synchronization (correlation coefficient close to 1), and this independently of the value of the injection rate. As already discussed in Figs. 1 and 5, the boundary between region I of good synchronization and region II of perfect synchronization also corresponds to the polarization switching boundaries. Importantly, we observe that Fig. 6 is consistent with the conclusion previously reported on polarization switching induced by optical injection of stationary light $[9,19]$, i.e., that the injected power required to induce the polarization switching of the slave laser decreases as the frequency detuning moves towards zero.

Figure 7 analyzes the mapping of the cross-correlation between slave laser LP mode intensities in the plane of the coupling parameters. As mentioned earlier the two slave laser LP mode intensities show some degree of anticorrelation in a large range of injection parameters inside region II, which also means that the slave laser $x$-LP mode shows some degree of antisynchronization with the injected master $x$-LP mode. Interestingly, the degree of anticorrelation (and therefore antisynchronization) increases as the injection rate decreases.

The synchronization quality inside region I (injectionlocking-type synchronization) can be significantly improved if we remove the parameter mismatch between master and slave laser linear cavity anisotropies, as shown in Fig. 8 for $\gamma_{a_{M}}=\gamma_{a_{S}}=-0.1, \gamma_{p_{M}}=\gamma_{p_{S}}=8.5 \mathrm{rad} / \mathrm{ns}$, all the other parameters remaining the same as in Fig. 6. Interestingly, for large injection strength the synchronization quality in region I can be as good as that in region II, which means that the two VCSELs synchronize well their LP mode dynamics whatever the frequency detuning between them. This result significantly differs from what is observed in comparable studies in edge-emitting lasers [45], where a good modal or total intensity synchronization occurs in relatively small parameter regions corresponding to injection-locking conditions, or to anticipative synchronization mechanisms. This result is here the consequence of the LP mode dynamics in VCSELs which
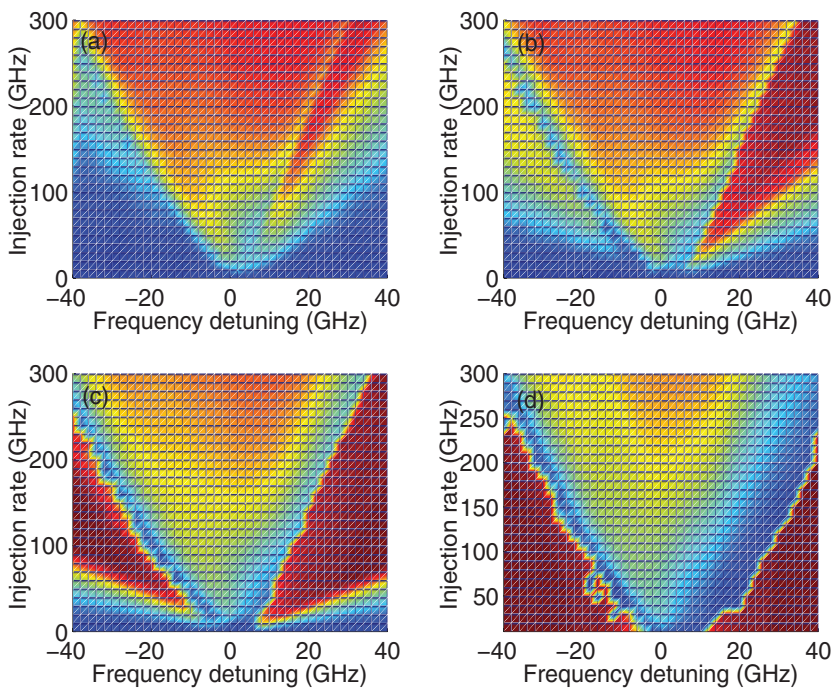

FIG. 9. (Color online) Same as Fig. 6 but with different values of the spin-flip relaxation rate parameter: (a) $\gamma_{s}=10 \mathrm{~ns}^{-1}$, (b) $\gamma_{s}$ $=30 \mathrm{~ns}^{-1}$, (c) $\gamma_{s}=40 \mathrm{~ns}^{-1}$, (d) $\gamma_{s}=300 \mathrm{~ns}^{-1}$.

makes it possible to observe two regions I and II in which good synchronization occurs.

\section{INFLUENCE OF $\gamma_{s}$ ON THE TWO REGIONS OF SYNCHRONIZATION}

We pay particular attention in this section to the influence of the spin-flip relaxation rate $\gamma_{s}$ on the results observed earlier. This parameter of the laser model accounts for microscopic processes that lead to the homogenization of the carrier spins. Several experiments have shown a good agreement with the SFM model when including a large or even infinite value for the $\gamma_{s}$ parameter [52], which also means that in such experiments the time scale for carrier spin relaxation processes is very short and hence does not play a role in the physics underlying polarization dynamics in VCSELs. By contrast, other experiments have resulted in a good fitting with the SFM model for a small value of $\gamma_{s}$ down to $\gamma_{s}$ $=10 \mathrm{~ns}^{-1}$ [53]. The spin-flip relaxation time scale becomes then comparable to other internal time scales of the VCSEL such as carrier relaxation time or inverse of birefringence frequency. In comparisons between numerical simulations performed with the SFM model and experiments it is therefore crucial to investigate the specific role of the $\gamma_{s}$ as a fitting parameter whose value may significantly vary from one VCSEL device to another one.

Figure 9 analyzes the mapping of correlation properties between $y$-LP mode intensity of the slave VCSEL and injected $x$-LP mode intensity of the master laser, for increasing values of $\gamma_{s}$ and all other parameters remaining the same as those used in Fig. 6. The color code for the mappings is the same as that used previously, hence making easier the comparison with previous mappings for different parameters. As shown in Fig. 9(d), increasing $\gamma_{s}$ up to $300 \mathrm{~ns}^{-1}$ does not significantly modify the picture of the mapping of the correlation coefficient. This also interestingly means that our results are valid even for VCSELs in which spin-flip relaxation 

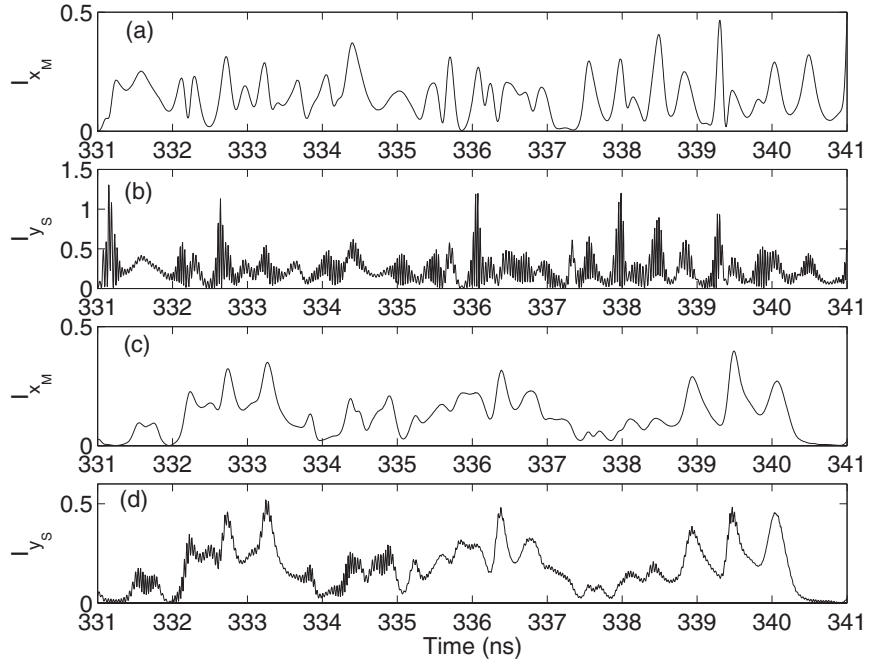

FIG. 10. Time traces of the $x$-LP master laser intensity and $y$-LP slave laser intensity for $\eta=200 \mathrm{GHz}, \Delta \omega / 2 \pi=30 \mathrm{GHz}$ and for two different values of the spin-flip relaxation rate parameter: (a), (b) $\gamma_{s}=10 \mathrm{~ns}^{-1}$ and (c), (d) $\gamma_{s}=40 \mathrm{~ns}^{-1}$.

mechanisms do not play a significant role in the physics of polarization switching. A decrease of $\gamma_{s}$, however, leads to interesting conclusions. The boundaries corresponding to region I, with single $y$-LP mode dynamics in the slave laser, are almost unaffected by a change in $\gamma_{s}$. However, the synchronization quality inside region I increases when the spinflip relaxation rate $\gamma_{s}$ decreases. By contrast, the comparison between Figs. 9(a)-9(c) and Fig. 6 shows that the decrease of $\gamma_{s}$ significantly influences the synchronization properties inside region II, where the normally depressed $y$-LP mode of the slave laser synchronizes with the injected $x$-LP mode of the master laser. For small values of $\gamma_{s}$ such as in (a) and (b) the synchronization quality between the master $x$-LP and slave $y$-LP modes in region II is now comparable or only slightly better than in region I. Additionally, region II of synchronization expands over small ranges of injection rates and frequency detuning and is located mostly in the positive frequency detuning side. For larger values of $\gamma_{s}$ such as in (c) and (d) the synchronization quality of region I is significantly smaller than that of region II and moreover, the region II of synchronization expands towards smaller injection rates and larger positive or negative frequency detunings. A region II of almost perfect synchronization quality between $y$-LP slave laser mode and $x$-LP master laser mode is therefore more easily observed in VCSELs with a relatively large $\gamma_{s}$ value.

The improvement of the synchronization quality in region II as $\gamma_{s}$ increases from the situation corresponding to Fig. 9(a) $\left(\gamma_{s}=10 \mathrm{~ns}^{-1}\right)$ to the situation corresponding to Fig. 9(c) $\left(\gamma_{s}=40 \mathrm{~ns}^{-1}\right)$, is made clear in Fig. 10. The time traces of the $x$-LP master laser intensity and of the $y$-LP slave laser intensity are plotted for these two values of $\gamma_{s}$. In the case of small $\gamma_{s}$ [Figs. 10(a) and 10(b)] the time traces exhibit a relatively good synchronization when looking at the envelopes of the pulses. The slave laser dynamics, however, exhibits more complex pulsing behavior with faster pulsating dynamics not present in the master laser dynamics. In the
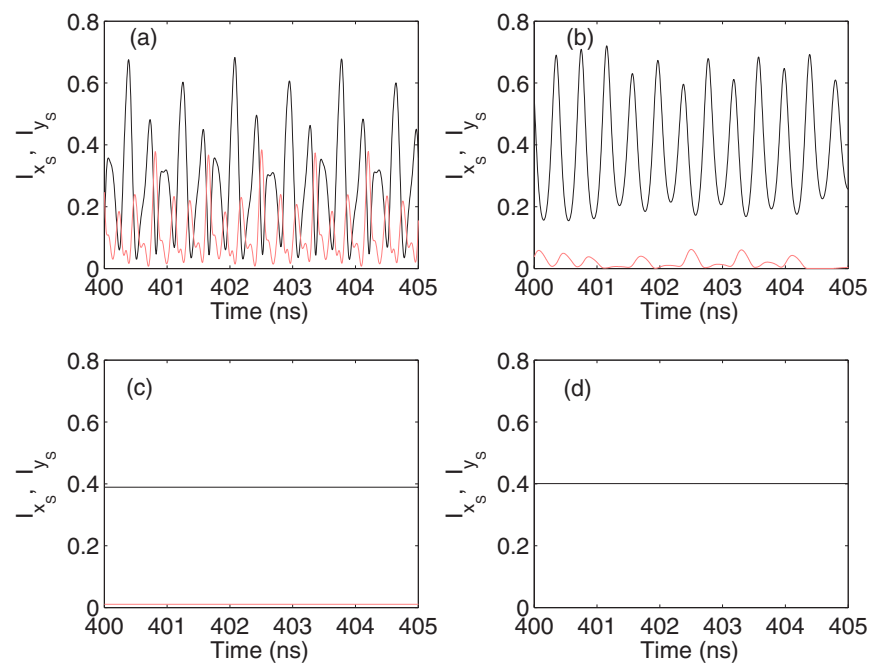

FIG. 11. (Color online) Time traces of the $x$-LP (black) and $y$-LP (red) slave laser mode intensities for different values of the spin-flip relaxation rate parameter: (a) $\gamma_{s}=10 \mathrm{~ns}^{-1}$, (b) $\gamma_{s}=30 \mathrm{~ns}^{-1}$, (c) $\gamma_{s}=40 \mathrm{~ns}^{-1}$, (d) $\gamma_{s}=300 \mathrm{~ns}^{-1}$.

case of larger $\gamma_{s}$ [Figs. 10(c) and 10(d)] the synchronization between the $x$-LP master laser intensity and the $y$-LP slave laser intensity is significantly improved and almost perfect.

The modifications of LP mode synchronization properties as the spin-flip relaxation rate varies also coincide with qualitative changes in the free-running slave laser LP mode dynamics, as better seen in Fig. 11. Figure 11 plots the slave laser LP mode intensity time traces when not subject to injection from the master laser, i.e., in the free-running operation, and for the values of $\gamma_{s}$ corresponding to the cases of Figs. 9(a)-9(d). In each case, the slave laser LP mode dynamics is such that the $x$-LP mode is the dominant LP mode, in agreement with our orthogonal optical injection scheme. In the case (d), as for $\gamma_{s}>50 \mathrm{~ns}^{-1}$, the $y$-LP mode is completely suppressed. However for smaller values of $\gamma_{s}$ the free-running slave VCSEL exhibits two-mode dynamics with either the LP mode intensities steady in time (c) or exhibiting a chaotic (b) or even a time-periodic dynamics (a). It is indeed well known from previous studies of the SFM model for a free-running VCSEL that the decrease of the value for the $\gamma_{s}$ parameter allows for stable elliptically polarized steady-state solution or time-dependent two mode dynamics [43]. Stable stationary elliptically polarized states and complex time-dependent two LP mode dynamics have been demonstrated experimentally in free-running VCSELs [54]. The results of Fig. 11 together with the corresponding synchronization results in Fig. 9 suggest that the difference of LP mode dynamics in the slave VCSEL, as a result of modifications of nonlinearities related to spin-flip relaxation mechanisms, strongly influences the synchronization properties in our orthogonally polarized master-slave injection scheme. More specifically, the existence of a strong, complex, two-mode dynamics in the free-running VCSEL [such as in Fig. 11(a)] seems to favor the existence of a large region I of good synchronization and a small region II of similar or slightly better synchronization quality. By contrast the only $x$-mode dynamics in the slave laser as obtained in 
Fig. 11(d) favors the existence of a large region II of perfect synchronization.

\section{CONCLUSIONS}

In summary, we have analyzed in detail the synchronization properties and polarization dynamics of two VCSELs unidirectionally coupled in a master-slave configuration with isotropic optical feedback on the master laser and orthogonal optical injection on the slave laser. The free-running slave laser is such that it emits only in $x$-LP mode and the injected light is polarized in the orthogonal, $y$-LP direction. Such a coupled VCSEL configuration has been analyzed in a recent experimental report [38]. Our results show interestingly two regions of different synchronization mechanisms. In the socalled region I the slave VCSEL switches its polarization to that of the injected light and emits only in a locked $y$-LP mode. The intensity of the $y$-LP slave laser mode is relatively well synchronized with the injected master $x$-LP mode, with a synchronization quality that strongly depends on the parameter mismatch between coupled VCSELs. In this region I the total intensity of the slave laser synchronizes to the total intensity of the master laser as well as the $y$-LP mode slave intensity synchronizes to the injected $x$-LP mode. In the socalled region II, by contrast, the slave VCSEL emits a twomode dynamics. The slave laser $y$-LP mode is almost perfectly synchronized with the injected $x$-LP mode, although with a significant attenuation. The slave laser $x$-LP mode is moreover anticorrelated with the slave laser $y$-LP mode, hence is antisynchronized with the injected master laser $x$-LP mode. Although the polarization modes exhibit an excellent synchronization, the total intensities of master and slave lasers are almost uncorrelated. The range of frequency detuning in which region II is found increases as the injection strength decreases, and moreover the boundaries of the region II are found to significantly depend on the $\gamma_{s}$ parameter which models the nonlinearity associated with spin-flip relaxation mechanisms in VCSELs. Both synchronization mechanisms, associated with regions I and II, are found to be robust against modifications of the device parameters, hence making their observations general to different VCSEL devices. Our results agree qualitatively with the synchronization properties found experimentally [38], but they also report on findings that stimulate further investigation. In particular the experiment reports only on the region II type of synchronization, and does not analyze its interesting dependency on the injection strength and internal device parameters, nor the synchronization properties of the total intensities. The theory predicts the existence of another synchronization region (region I), which also behaves very differently with respect to the total intensity synchronization, robustness on parameter mismatch, and influence of spin-flip relaxation rate.

\section{ACKNOWLEDGMENTS}

The authors acknowledge the support of the Conseil Régional of Lorraine and of the COST 288 European Action. This work was supported in part by BELSPO IAP 6/10, by FWO-Vlaanderen, OZR-VUB for the GOA and IOF projects. The authors would like to thank D. S. Citrin for his fruitful comments.
[1] K. Iga, IEEE J. Sel. Top. Quantum Electron. 6, 1201 (2000).

[2] C. J. Chang-Hasnain, J. P. Harbison, G. Hasnain, A. C. Von Lehmen, L. T. Florez, and N. G. Stoffel, IEEE J. Quantum Electron. 27, 1402 (1991).

[3] K. D. Choquette, R. P. Schneider, and K. L. Lear, IEEE J. Sel. Top. Quantum Electron. 1, 661 (1995).

[4] M. San Miguel, in Semiconductor Quantum Electronics: From Quantum Physics to Smart Devices, edited by A. Miller (Institute of Physics, Bristol, 1999).

[5] K. Panajotov et al., in Nanoscale Linear and Nonlinear Optics, edited by M. Bertolotti, C. M. Bowden, and C. Sibilia (American Institute of Physics, Melville, NY, 2001).

[6] A. Valle, L. Pesquera, and K. A. Shore, IEEE Photonics Technol. Lett. 10, 639 (1998).

[7] A. Tabaka, M. Peil, M. Sciamanna, I. Fischer, W. Elsäßer, H. Thienpont, I. Veretennicoff, and K. Panajotov, Phys. Rev. A 73, 013810 (2006).

[8] M. Sciamanna, Ph.D. dissertation, 2004.

[9] Z. G. Pan, S. Jiang, M. Dagenais, R. A. Morgan, K. Kojima, M. T. Asom, R. E. Leibenguth, G. D. Guth, and M. W. Focht, Appl. Phys. Lett. 63, 2999 (1993).

[10] A. Valle, L. Pesquera, S. I. Turovets, and J. M. Lopez, Opt. Commun. 208, 173 (2002).

[11] M. Sciamanna, A. Valle, P. Mégret, M. Blondel, and K. Pana- jotov, Phys. Rev. E 68, 016207 (2003).

[12] C. Masoller and N. B. Abraham, Phys. Rev. A 59, 3021 (1999).

[13] M. Giudici, S. Balle, T. Ackemann, S. Barland, and J. R. Tredicce, J. Opt. Soc. Am. B 16, 2114 (1999).

[14] M. Sciamanna, C. Masoller, N. B. Abraham, F. Rogister, P. Mégret, and M. Blondel, J. Opt. Soc. Am. B 20, 37 (2003).

[15] M. Sciamanna, C. Masoller, F. Rogister, P. Mégret, N. B. Abraham, and M. Blondel, Phys. Rev. A 68, 015805 (2003).

[16] M. Sondermann, H. Bohnet, and T. Ackemann, Phys. Rev. A 67, 021802(R) (2003).

[17] M. Sciamanna, K. Panajotov, H. Thienpont, I. Veretennicoff, P. Mégret, and M. Blondel, Opt. Lett. 28, 1543 (2003).

[18] Y. Hong, K. A. Shore, A. Larsson, M. Ghisoni, and J. Halonen, Electron. Lett. 36, 2019 (2000).

[19] I. Gatare, M. Sciamanna, J. Buesa, H. Thienpont, and K. Panajotov, Appl. Phys. Lett. 88, 101106 (2006).

[20] J. Buesa, I. Gatare, K. Panajotov, H. Thienpont, and M. Sciamanna, IEEE J. Quantum Electron. 42, 198 (2006).

[21] F. Robert, P. Besnard, M. L. Charès, and G. Stéphan, IEEE J. Quantum Electron. QE-33, 2231 (1997).

[22] H. Li, A. Hohl, A. Gavrielides, H. Hou, and K. D. Choquette, Appl. Phys. Lett. 72, 2355 (1998).

[23] M. Sciamanna, T. Erneux, F. Rogister, O. Deparis, P. Mégret, 
and M. Blondel, Phys. Rev. A 65, 041801(R) (2002).

[24] M. Sciamanna, F. Rogister, O. Deparis, P. Mégret, M. Blondel, and T. Erneux, Opt. Lett. 27, 261 (2002).

[25] P. Colet and R. Roy, Opt. Lett. 19, 2056 (1994).

[26] G. D. Van Wiggeren and R. Roy, Science 279, 1198 (1998).

[27] G. D. Van Wiggeren and R. Roy, Phys. Rev. Lett. 81, 3547 (1998).

[28] C. R. Mirasso, P. Colet, and P. Garcia-Fernandez, IEEE Photonics Technol. Lett. 8, 2999 (1996).

[29] R. Roy and K. S. Thornburg, Phys. Rev. Lett. 72, 2009 (1994).

[30] T. Sugawara, M. Tachikawa, T. Tsukamoto, and T. Shimizu, Phys. Rev. Lett. 72, 3502 (1994).

[31] J.-P. Goedgebuer, L. Larger, and H. Porte, Phys. Rev. Lett. 80, 2249 (1998).

[32] S. Sivaprakasam and K. A. Shore, Opt. Lett. 24, 1200 (1999).

[33] S. Tang and J. M. Liu, Opt. Lett. 26, 1843 (2001).

[34] A. Argyris, D. Syvridis, L. Larger, V. Annovazi-Lodi, P. Colet, I. Fischer, J. Garcia-Ojalvo, C. R. Mirasso, L. Pesquera, and K. A. Shore, Nature (London) 438, 343 (2005).

[35] P. S. Spencer, C. R. Mirasso, P. Colet, and K. A. Shore, IEEE J. Quantum Electron. 34, 1673 (1998).

[36] P. S. Spencer and C. R. Mirasso, IEEE J. Quantum Electron. 35, 803 (1999).

[37] R. Ju, P. S. Spencer, and K. A. Shore, IEEE J. Quantum Electron. 41, 1641 (2005).

[38] Y. Hong, M. W. Lee, P. S. Spencer, and K. A. Shore, Opt. Lett. 29, 1215 (2004).

[39] N. Fujiwara, Y. Takiguchi, and J. Ohtsubo, Opt. Lett. 28, 1677 (2003).

[40] M. W. Lee, Y. Hong, and K. A. Shore, IEEE Photonics Tech- nol. Lett. 16, 2392 (2004).

[41] R. Vicente, T. Perez, and C. R. Mirasso, IEEE J. Quantum Electron. 38, 1197 (2002).

[42] D. W. Sukow, A. Gavrielides, T. McLachlan, G. Burner, J. Amonette, and J. Miller, Phys. Rev. A 74, 023812 (2006).

[43] J. Martin-Regalado, F. Prati, M. San Miguel, and N. B. Abraham, IEEE J. Quantum Electron. 33, 765 (1997).

[44] C. R. Mirasso, R. Vicente, P. Colet, J. Mulet, and T. Perez, C. R. Phys. 5, 613 (2004).

[45] A. Locquet, C. Masoller, and C. R. Mirasso, Phys. Rev. E 65, 056205 (2002).

[46] A. Locquet, F. Rogister, M. Sciamanna, P. Megret, and M. Blondel, Phys. Rev. E 64, 045203(R) (2001).

[47] A. Locquet, C. Masoller, P. Megret, and M. Blondel, Opt. Lett. 27, 31 (2002).

[48] F. Rogister, A. Locquet, D. Pieroux, M. Sciamanna, O. Deparis, P. Megret, and M. Blondel, Opt. Lett. 26, 1486 (2001).

[49] C. Masoller, Phys. Rev. Lett. 86, 2782 (2001).

[50] K. Panajotov, B. Nagler, G. Verschaffelt, A. Georgievski, H. Thienpont, J. Danckaert, and I. Veretennicoff, Appl. Phys. Lett. 77, 1590 (2000).

[51] J. Revuelta, C. R. Mirasso, P. Colet, and L. Pesquera, IEEE Photonics Technol. Lett. 14, 140 (2002).

[52] P. Besnard, M.-L. Charés, G. Stéphan, and F. Robert, J. Opt. Soc. Am. B 16, 1059 (1999).

[53] M. Sondermann, M. Weinkath, T. Ackemann, J. Mulet, and S. Balle, Phys. Rev. A 68, 033822 (2003).

[54] T. Ackemann and M. Sondermann, Appl. Phys. Lett. 78, 3574 (2001). 